\title{
Local networks in visual cortex and their influence on neuronal responses and dynamics
}

\author{
James Schummers, Jorge Mariño ${ }^{1}$, Mriganka Sur * \\ Department of Brain and Cognitive Sciences, Picower Center for Learning and Memory, MIT, E25-235, 45 Carleton Street, \\ Cambridge, MA 02139, United States
}

\begin{abstract}
Networks of neurons in the cerebral cortex generate complex outputs that are not simply predicted by their inputs. These emergent responses underlie the function of the cortex. Understanding how cortical networks carry out such transformations requires a description of the responses of individual neurons and of their networks at multiple levels of analysis. We focus on orientation selectivity in primary visual cortex as a model system to understand cortical network computations. Recent experiments in our laboratory and others provide significant insight into how cortical networks generate and maintain orientation selectivity. We first review evidence for the diversity of orientation tuning characteristics in visual cortex. We then describe experiments that combine optical imaging of orientation maps with intracellular and extracellular recordings from individual neurons at known locations in the orientation map. The data indicate that excitatory and inhibitory synaptic inputs are summed across the cortex in a manner that is consistent with simple rules of integration of local inputs. These rules arise from known anatomical projection patterns in visual cortex. We propose that the generation and plasticity of orientation tuning is strongly influenced by local cortical networks - the diversity of these properties arises in part from the diversity of neighbourhood features that derive from the orientation map.
\end{abstract}

(c) 2005 Elsevier Ltd. All rights reserved.

Keywords: Visual cortex; Orientation tuning; Cortical maps; Optical imaging; Intracellular recording; Response dynamics

\section{Introduction}

Cortical neurons are embedded in a dense network composed of myriad connections arising from a number of sources. Over a century of work has contributed to a relatively detailed description of the wiring rules that govern the thousands of synaptic connections that impinge on individual cortical neurons. The cortical microcircuit in primary visual cortex (V1) is now understood at a reasonable level of detail as a result of these efforts [18,31], though much still remains to be resolved. In particular, issues regarding the cell-type specificity of connections and the

\footnotetext{
${ }^{*}$ Corresponding author.

E-mail address: msur@mit.edu (M. Sur).

${ }^{1}$ Present address: Neuroscience and Motor Control Group (Neurocom), Univ. A Coruña, Fac. CC. da Saúde, Campus de Oza, 15006 A Coruña, Spain.
}

degree to which particular cortical circuits are specialized remain topical issues [41,47]. There is also a vast literature describing the response properties of individual neurons in V1 electrophysiologically. Starting with the original description of orientation selectivity over 40 years ago [32], the response selectivity of V1 neurons to a large number of visual features have been examined. Yet, a substantial gap remains between the description at a network level and a functional description of the behavior of individual neurons at the electrophysiological level. The majority of our understanding of the functional properties of cortical neurons comes from single cell recordings with extracellular electrodes. However, most studies of the response properties of cortical neurons in vivo are blind to the position in the cortical circuit in which they sit. Thus, a dichotomy exists between the functional description of individual neurons and a structural description of cortical networks. New approaches are required that allow simultaneous 
characterization of the functional response properties of individual neurons and the nature of the cortical network in which they are located. We will describe recent work aimed at bridging this gap.

\subsection{Orientation tuning in primary visual cortex}

The primary visual cortex (V1) provides an excellent model system to study the behavior of cortical networks. V1 is amenable to study with a number of techniques and many of the basic properties of the cells and their connections are well described. The property of orientation selectivity is perhaps the most thoroughly studied response property of V1 neurons. The majority of V1 neurons are selective to the orientation of a stimulus pattern presented in their receptive fields. This is often termed an emergent property, because the major source of afferent drive to V1, the lateral geniculate nucleus of the thalamus (LGN), contains neurons that are relatively unselective for stimulus orientation. However, it is not entirely clear to what extent this transformation is strictly emergent. A large body of work over the last several decades has investigated the mechanisms that generate orientation selectivity in LGN-recipient layer IV neurons in V1. There has been considerable controversy regarding the degree to which it is the precise arrangement of the "feedforward" thalamocortical projections that converge to create orientation tuning in neurons in layer IV of V1. There is now substantial experimental support for some of the aspects of this model ([24,37,50]; see [25] for a recent review). However, there are also numerous features of orientation tuning that this model cannot explain $([14,21,44,52]$; see $[63,65]$ for a review). Several computational models have proposed alternate schemes, based primarily on recurrent amplification of inputs to cortex, that can largely explain the experimental data without recourse to a strict arrangement of thalamocortical projections required by the feedforward model $[7,17,64]$. Some models that rely entirely on intracortical connections to produce tuning can produce orientation selectivity without any bias in the thalamocortical input [22]. As the specifics regarding this receptive field transformation are resolved $[25,63,65]$, other issues regarding orientation tuning have begun to emerge into the spotlight.

\subsection{Generation vs. maintenance of tuning}

One issue that we feel has been underemphasized is the maintenance of orientation selectivity. Regardless of the particular mechanisms that generate the initial selectivity in layer IV simple cells, the remaining inputs to those simple cells, as well as all of the inputs to neurons outside of the input layers, must be carefully coordinated to ensure that selectivity is maintained in the responses of neurons within the column. Given the large number of inputs that any given neuron receives, from a distributed network of neurons, this is not a trivial issue. The percentage of drive provided by thalamocortical inputs is estimated to be roughly a third of the total in simple cells [25]. The rest of the synaptic drive to simple cells derives from the cortex. In fact, considering the multitude of other stimulus features that are computed by V1 neurons [33], as well as the modulatory and feedback projections that V1 receives, it is impressive that V1 neurons are generally so sharply tuned at all.

The issue of the generation of orientation tuning is strictly limited to the emergence of tuning in layer IV simple cells, and yet there are relatively few studies that carefully limit the recordings to these neurons. (Note: we are largely limiting our description to the cat, where the majority of relevant work has been done. There are substantial differences in the laminar organization of orientation selectivity in other species, such as the ferret, monkey and tree shrew.) Much of the evidence that has been put forth in support of recurrent models of orientation tuning comes from experiments not restricted to layer IV simple cells. For instance, it has been demonstrated that remote inactivation of orthogonal orientation columns can reduce the orientation selectivity of neurons located $350-700 \mu \mathrm{m}$ distant $[13,15]$. This provides strong support for the importance of an intact cortical network for normal orientation tuning. A substantial portion of these data were taken from layer II/III complex cells, and thus in our view, are more relevant to the question of the maintenance of orientation tuning. Even in simple cells, such data do not rule out a feedforward mechanism for the initial generation of tuning, but they do emphasize the importance of interactions within the local network in shaping the responses of cortical neurons. Another argument for recurrent mechanisms of orientation tuning is the complex temporal dynamics of responses in macaque V1 neurons [51,52]. However, it is important to note that complex dynamics are not so evident in layer IVc, and therefore cannot argue for or against the feedforward model, but this behavior does hint that the cortical interactions may be shaping response tuning in interesting and complex ways.

Regardless of the particular mechanism that generates orientation tuning in layer IV simple cells, the maintenance of orientation tuning is necessarily cortical. We take the view that this is not a trivial problem, and it is one that deserves further attention. Experiments are needed to define the cortical inputs involved in the process and the rules by which they are integrated.

\section{Diversity of orientation tuning properties}

A growing number of studies have emphasized the diversity of orientation tuning properties of V1 neurons. Although it has long been recognized that the orientation tuning characteristics of all V1 neurons are not identical, most models of orientation tuning do not explicitly take account of this fact. There are a number of obvious sources of variability among V1 neurons, including laminar 
position, receptive field type and position in the orientation preference map. It is only recently that these parameters are being considered in evaluating orientation selectivity.

\subsection{Diversity in subthreshold tuning}

Several recent reports have described diversity in several response parameters related to orientation tuning. Early intracellular recordings of subthreshold membrane potential suggested that the contribution of inhibition to shaping the orientation tuning curve was variable $[48,70]$. Many neurons showed inhibition tuned to the same orientation as the excitatory and spike response tuning curves. However, other neurons showed clear hyperpolarization only at or near the orthogonal orientation. This work led to the hypothesis that more than one mechanism may produce orientation tuning in V1 neurons [69]. Other studies have failed to find substantial evidence for orthogonal tuning of inhibition $[1,23,46]$. Recently, in a comprehensive study, Monier et al. [44] have convincingly demonstrated that indeed the orientation tuning of inhibitory synaptic inputs shows substantial variability across the population. While the majority of neurons receive inhibition that peaks at their preferred orientation, a substantial minority receive inhibitory inputs that are orthogonal or oblique to their preferred orientation. The authors hypothesized that this diversity in synaptic mechanisms responsible for orientation tuning might be related to the diversity in the local cortical circuitry that results from the inhomogeneity of the orientation preference map. In fact, it has recently been shown that there are substantial differences in the synaptic inputs to neurons that depend strongly on their location within the orientation preference map [60]. Evidence suggesting that the diversity of local circuits generated by the orientation preference map can strongly influence the synaptic responses of V1 neurons will be discussed in detail below.

Another source of diversity in the inputs to V1 neurons is their laminar position. The fairly stereotypical pattern of synaptic connections between different layers $[18,41]$ leads to differences in the sources of input to neurons in different cortical layers. Although it has long been recognized that laminar differences exist [26,38], many studies have not reported the laminar location of recorded cells. Several recent reports have reexamined the influence of laminar position on orientation properties of V1 neurons. Martinez et al. [42] recorded intracellularly from neurons from all cortical layers and reconstructed their morphology. Interestingly, they found that there are laminar-specific differences in the synaptic mechanisms that compute orientation tuning. Specifically, they found that neurons in layer $\mathrm{V}$ have inhibitory tuning that is orthogonal to the preferred orientation of the cell. Throughout the other cortical layers, inhibition tended to be iso-oriented to excitation. There is also evidence that the influence of inhibition on orientation tuning in monkey V1 depends strongly on layer. Sato et al. [57] found that the broadening of orientation tuning that results from local iontophoresis of GABA antagonists was strongest outside of the input layers. Thus, orientation tuning may be re-computed in different cortical layers, by use of different synaptic integration mechanisms.

\subsection{Diversity in spike tuning}

Several recent studies have highlighted the diversity of several aspects of orientation tuning in V1 neurons. Ringach and colleagues [53] have carefully studied the orientation tuning properties of a large set of monkey V1 neurons with known laminar position. They found that orientation selectivity varies systematically with laminar location, but that there is substantial spread of selectivity in any given lamina as well. Overall, they found an even distribution of cells across the spectrum of selectivity, from extremely sharp tuning, to completely unselective. This work highlights the problems that can arise from assuming that a single model can explain the orientation tuning of all V1 neurons.

Further work from the same group has examined the temporal dynamics of orientation tuning using reverse-correlation analysis of the responses to dynamic grating stimuli [52]. Using this approach, it is possible to examine suppression and enhancement of firing on a millisecond time scale for each orientation. The authors found evidence for tuned enhancement and suppression, as well as untuned, global, enhancement and suppression, with each component following a different time course. Interestingly, they only found tuned suppression in a subpopulation of cells, again suggesting different mechanisms acting to produce sharp tuning in different cells.

Another intriguing example of diversity related to orientation tuning is in the input-output transformation performed by V1 neurons. Volgushev et al. [73] compared the tuning curves of the subthreshold membrane depolarization and the spiking response in a population of V1 neurons. They found that the degree of sharpening in this transformation varied over a large range. Many neurons had already sharp membrane potential tuning curves, whereas others had broad input tuning, which was dramatically sharpened by the transformation to spikes. Carandini et al. [12] have found similar results.

These studies make it clear that a single standard model cannot provide a complete description of orientation tuning in V1. There is diversity in the spike tuning shape and dynamics, synaptic contributions of excitation and inhibition to tuning, as well as the input-output transformation performed by individual neurons. Providing an explanation for the diversity in all these aspects of the computation of orientation tuning will be an important goal for future conceptions of orientation tuning in V1. A logical assumption is that this diversity reflects a diversity of local circuit architectures, and can therefore provide an opportunity to link circuitry with function. 


\section{Orientation map and anatomical connectivity}

The representation of orientation in V1 consists of an orderly, latticed map of preferred orientation. Columns of neurons sharing similar orientation preference are grouped into orientation domains, which are arranged radially around pinwheel centers, where orientation domains representing all orientations converge $[8,9]$. The pinwheel centers are spaced semi-regularly, with separations typically $600-800 \mu \mathrm{m}$ [49]. Thus, over large expanses of V1, preferred orientation changes smoothly and gradually, but at pinwheel centers, there is an abrupt shift in preferred orientation over an extremely small scale. These maps provide an important link between the activity of individual neurons and the functional properties of the entire network surrounding them.

There are clear links between the functional architecture and the anatomical architecture. The long-range patchy connections of superficial layer neurons preferentially link domains representing similar orientations [29,54]; bulk injection of a retrograde tracer into an orientation domain will label neurons in other orientation domains representing similar orientations, although the degree of selectivity of these connections is debatable [36]. Thus, it appears that there may be a structural basis for the orientation map layout in the form of the long-range intrinsic connections. Interestingly, when an orientation map is artificially induced in auditory cortex by early postnatal rewiring, the horizontal connections in the rewired auditory cortex take on somewhat patchy patterns [62]. There is also intriguing recent data that these horizontal connections, and by extension the map they represent, may influence the spontaneous patterns of cortical activity $[4,68]$. There is also evidence in tree shrews and monkeys that the extent of the patchy connections may be extended along the retinotopic dimension that reflects the orientation that they represent [11].

Thus, the evidence that the pattern of long-range connections reflects the structure of the orientation map is fairly strong. However, the retinotopic space represented by the long-range connections impinging on any given position most likely falls outside of the classical receptive field for that location $[27,66]$. Hence, the long-range connections probably only mediate surround influences on the receptive field, which is driven by more local inputs with overlapping retinotopic representations. Though the range of the retinotopic representation of the long-range connections relative to different metrics of receptive field size is under debate [2], the computation of orientation tuning per se is most likely mediated by local connections representing only the very center of the receptive field. The evidence that these local connections respect the structure of the orientation map is generally lacking. Bulk tracer injections label a dense, homogeneous set of neurons within $\sim 500 \mu \mathrm{m}$ of the injection site [11,28,36]. Individual filled neurons have dendritic arbors that can be seen to cross the boundaries of orientation domains and pinwheel cen- ters [39]. Furthermore, injections targeted to pinwheel centers label an isotropic region comparable to that labeled with injections into orientation domains [76]. There is no evidence for anisotropy, or other specific targeting of local connection patterns to maintain the orientation specificity of these connections, as there is for long-range connections. Hence, based on anatomical data, it would be expected that neurons near pinwheel centers receive much more broadly tuned synaptic inputs from the local circuit.

Physiological experiments also support this architecture. Cross-correlation experiments suggest that nearby neurons are most likely to be synaptically coupled, even near pinwheel centers $[16,61]$. In an elegant series of combined optical imaging and slice recording experiments, it has been demonstrated that the synaptic input to ferret V1 neurons arises from an isotropic input region [55,56,74]. Numerically, the vast majority of input comes from a region within $\sim 500 \mu \mathrm{m}$ of the recorded cell. The spatial distribution and scale of integration is fairly similar for inhibitory and excitatory inputs. For neurons located in orientation domains, the result is reasonably sharp tuning of local inputs, with the inhibitory inputs being slightly more broadly tuned. Unfortunately, neurons were not recorded near pinwheel centers, so the anatomical demonstration of unselective inputs to pinwheel centers remains to be confirmed in this paradigm. We would expect, based on the tracer injection and the cross-correlation results, that neurons recorded near pinwheel centers would respond to stimulation of sites representing all orientations within $\sim 500 \mu \mathrm{m}$.

It appears, therefore, that there are dense connections between neighboring sites in V1, without regard for the functional representation of orientation. Thus, neurons situated close to pinwheel centers are likely to be connected with neurons having all preferred orientations, whereas neurons located in orientation domains are connected only with neurons sharing similar orientation preferences.

\section{Orientation tuning relative to orientation map}

The orientation preference map provides an excellent opportunity to study the influence of local circuits on orientation tuning. Based on the spatial rules of connectivity extracted from the anatomical and physiological experiments described in the previous section, it is possible to make a reasonable estimation of the orientation composition of the local inputs to any location in the orientation map. Fig. 1A shows a representation of the estimated regions over which two example neurons, one situated at a pinwheel center, and one in the center of an orientation domain, would be expected to pool local inputs. Fig. 1B and $\mathrm{C}$ shows two examples of such an analysis. For a location at a pinwheel center (Fig. 1A left circle, Fig. 1B) and in an orientation domain (Fig. 1A right circle, Fig. 1C), the local input tuning was estimated by calculating a histogram of pixels representing each orientation within a circle of radius $400 \mu \mathrm{m}$. The estimated local input tuning is nearly 
A

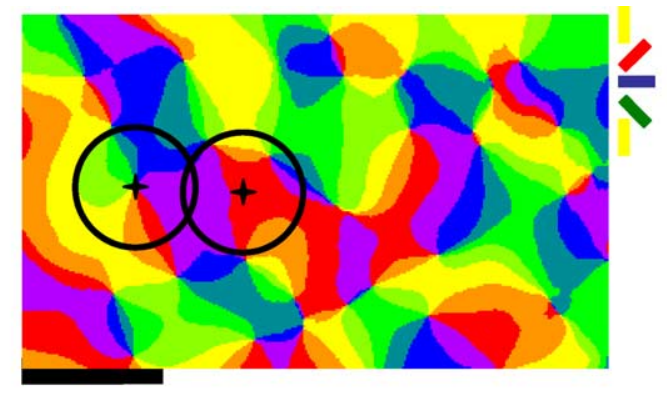

B

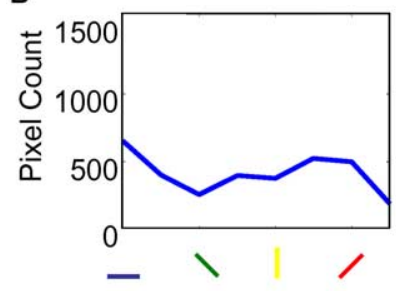

D

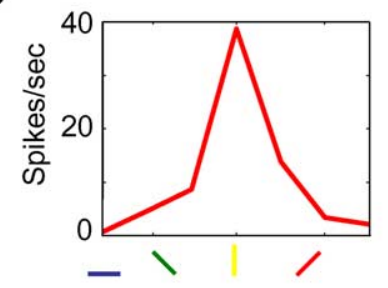

C

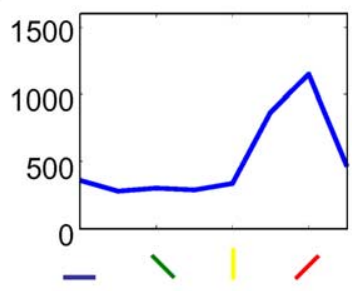

E

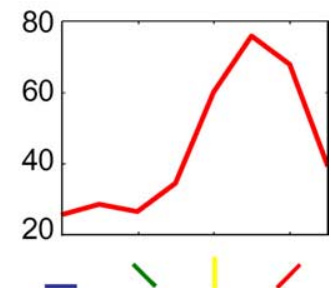

Fig. 1. Neurons near pinwheel centers are sharply orientation tuned, despite the broadly tuned inputs arising from the local network. A Orientation preference map obtained from cat area 17 with optical imaging of intrinsic signals. The preferred orientation of each pixel is binned into eight orientations ( $22.5^{\circ}$ interval), and represented by the pseudocolor code indicated by the legend to the right of the map. The spatial scale is indicated by the black bar, which represents $1 \mathrm{~mm}$ of cortical distance. The two circles represent estimations of the spatial extent of the dense local projections to the points at the center of each circle, marked by crosses. B,C. Histograms of the number of pixels representing each orientation that fall within the two circles shown in A, color coded according to the same code as in panel A (shown below the plot). Panel B is the histogram calculated from the left circle in Panel A, centered on a pinwheel center, and therefore the orientation distribution is flat. Panel C is calculated from the circle centered on an orientation domain, resulting in a peaked distribution, centered on the orientation represented in the orientation domain. D,E. Orientation tuning curves measured with extracellular recordings from the two locations marked by crosses in panel A. The stimulus orientations are again coded with the same color code as in panels A-C for ease of comparison. Both cells are well tuned for orientation, despite the differences in the surrounding orientation representations estimated from the orientation map.

flat for the pinwheel center, and sharply tuned for the orientation domain location. Surprisingly, studies that have measured the orientation tuning characteristics of neurons near and far from pinwheel centers have failed to find any differences in their tuning curves [19,40,60]. Fig. 1D-E show the firing rate tuning curves of neurons recorded at each of the two sites indicated in Fig. 1A. The tuning curves are fairly similar, representative of the results from large populations of recordings. This result presents an interesting conundrum: neurons are sharply tuned even

when the most likely source of the majority of their synaptic inputs are broadly tuned.

\subsection{Orientation tuning of subthreshold responses depends on map location}

In order to explore the influence of the local cortical circuit on the responses of V1 neurons, we have combined optical imaging of intrinsic signals with whole cell patch recordings in vivo. This approach allows the features of the responses of individual neurons to be correlated with a precise description of the orientation representation in the patch of cortex surrounding them. Unlike previous experiments combining extracellular recordings with optical imaging, the use of intracellular recordings permits the measurement of subthreshold membrane potential, which is much more closely linked to synaptic inputs than the spiking activity of neurons. Anatomical data suggests that a large majority of the synaptic input to $\mathrm{V} 1$ neurons is derived from a fairly small patch of surrounding cortex $[11,76]$. We are therefore in a position to test whether the tuning of the subthreshold membrane potential responses reflects the orientation representation in the local neighborhood.

Fig. 2 shows the responses of a simple cell located in an orientation domain in response to stimulation with drifting gratings spanning $180^{\circ}$ in orientation. Panel A shows the membrane potential traces in response to each of eight orientations and a blank screen. At the preferred orientation, there is a depolarizing response that is modulated at the temporal frequency of the grating drift. There is also a DC offset during the entire duration of the grating presentation on which the modulated component sits. There is a weaker response to near preferred orientations, and at the orthogonal orientation, there is almost no response at all. Panel B shows the spike response of the cell. The cell is sharply tuned-there is no response more than $22^{\circ}$ from the preferred orientation. The spike response is very strongly modulated by the grating phase, typical of simple cells. Panel $\mathrm{C}$ shows the tuning curves computed from the DC component of the membrane potential and spike responses to the full $360^{\circ}$ of stimulus presented.

Fig. 3 shows the responses of a simple cell located near a pinwheel center. The pattern of responses is clearly different from that of the cell shown in Fig. 2. The response to the preferred orientation is fairly similar - there is a mean depolarization of roughly $10 \mathrm{mV}$ that shows clear modulation with the grating phase. However, there is also a strong response all orientations, including the orthogonal. The depth of the modulated component is much weaker or non-existent, but the DC component of the response is nearly as strong at the orthogonal as at the preferred. This cell appears to be receiving strong synaptic drive regardless of the stimulus orientation. The spike responses, in contrast, are restricted to the preferred orientations.

Fig. 4 demonstrates the differences in the tuning of subthreshold depolarization in neurons situated near to, or far from, pinwheel centers. Fig. 4A shows the average firing 

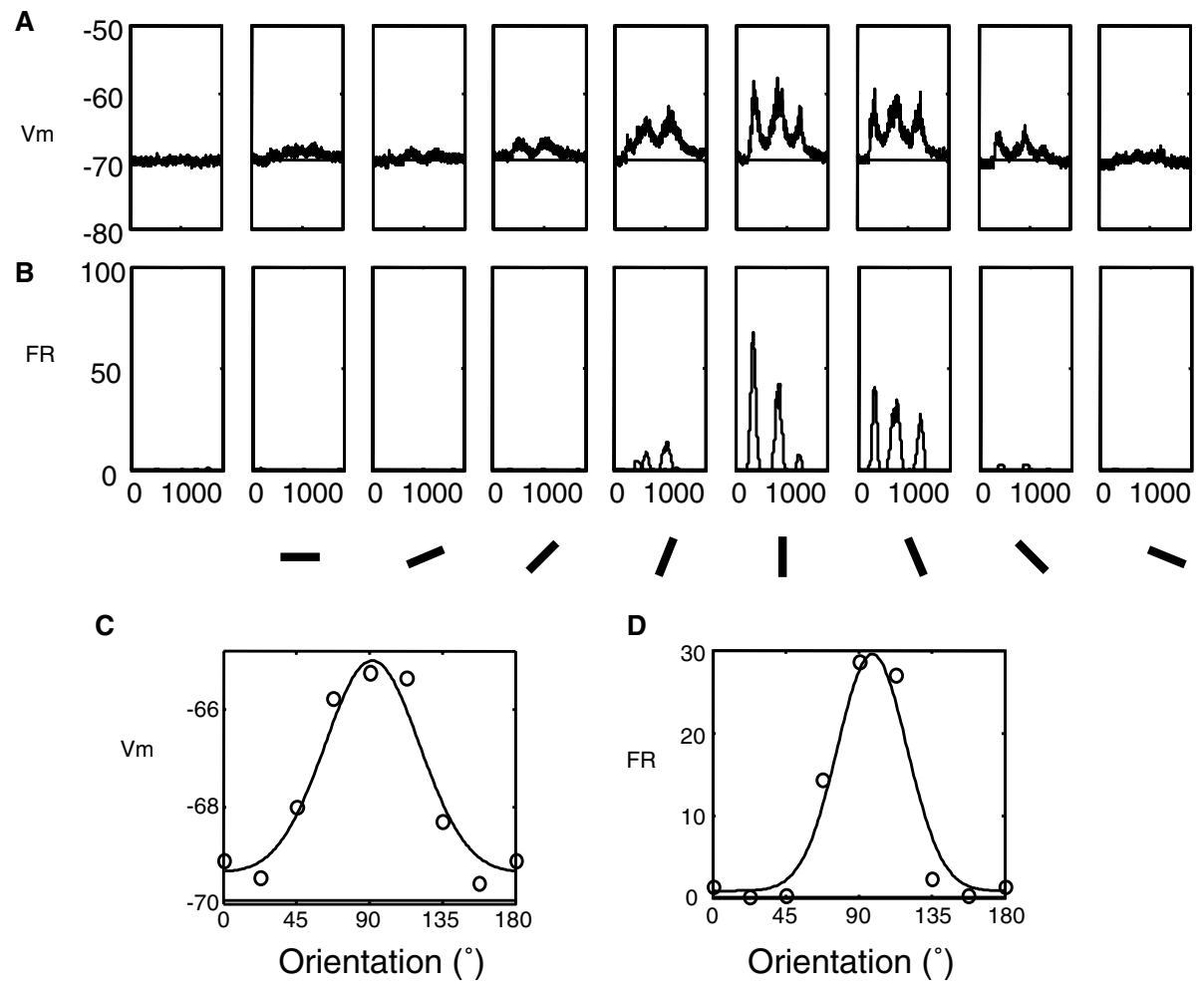

D

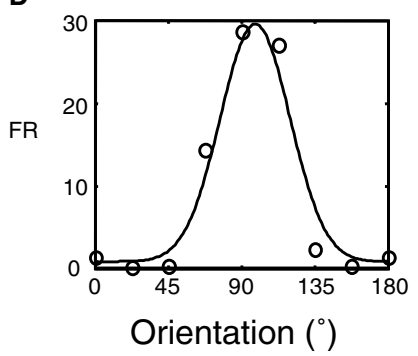

Fig. 2. Membrane potential and firing rate tuning of a simple cell in an orientation domain. A. Membrane potential traces during stimulation with each of eight stimulus orientations, centered on the preferred orientation of the cell. Each trace is the average of five trials after removal of spikes. The thin line represents the resting membrane potential, in the absence of visual stimulation. The panel on the far left is the membrane potential in response to a blank stimulus. B. PSTH plot of the spike response, extracted from the membrane potential responses in A. The time scale in B also applies to A. C. Tuning curve of the mean membrane potential $(\mathrm{Vm})$ in response to each stimulus orientation. The open circles represent measured data points; continuous line is the Gaussian fit to the data. D. Tuning curve of the spike firing rate (FR). Conventions as in C. Horizontal lines represent resting potential in C and spontaneous FR in D.

rate tuning curves of neurons located near pinwheel centers (dashed line), and those located far from pinwheel centers, in orientation domains (solid line). As previously demonstrated [40], the tuning curves are nearly indistinguishable. These population averages include neurons located in all cortical layers, and with both simple and complex receptive field types. Thus, other potential sources of diversity have probably been averaged out. As shown in Fig. 4B, there is a dramatic difference in the subthreshold tuning curves for neurons located near pinwheel centers. The difference is most prominent on the flanks of the tuning curves, near the orthogonal orientation. Neurons near pinwheel centers show a response that is almost twice as large as the responses of neurons in orientation domains. Furthermore, the response to the orthogonal orientation is almost half of the amplitude of the response to the preferred orientation. This difference is not at all apparent in the firing rate tuning curves, indicating that the large depolarizations at non-preferred orientations are filtered out by the spike threshold. Thus, V1 neurons appear to receive synaptic input that reflects the local cortical circuit composition, but the inputs are transformed differently to produce location invariant spike tuning.

The results of this experiment suggest that the subthreshold membrane potential responses of V1 neurons are correlated with the composition of the local patch of cortex surrounding them. In fact there is a clear linear relationship between the selectivity of the orientation representation in the local neighborhood and the selectivity of the membrane potential response [60]. Furthermore, the modulation of the membrane potential response between the preferred and orthogonal orientation is also strongly correlated with the orientation representation in the local neighbourhood of cells. This study used the same procedure illustrated in Fig. 1 to estimate the local input tuning. Thus, a simple model of local input pooling can capture the majority of subthreshold tuning in V1 neurons.

It is worth noting that the calculation of response tuning by the Orientation Selectivity Index [60] includes the DC component of the response. The F1 (temporally modulated component) to F0 (DC component) ratio of the spike response is used to classify cells as simple or complex, but the F1 component of the membrane response in simple cells does not match the local input tuning particularly well in all cells. The DC component is the part of the response that is not specific for spatial phase of the stimulus. This raises the possibility that the local circuit contribution to the subthreshold response is not phase selective, as proposed in some models of cortical circuits [43], but not others [67]. It is also possible that the orientation map used to 

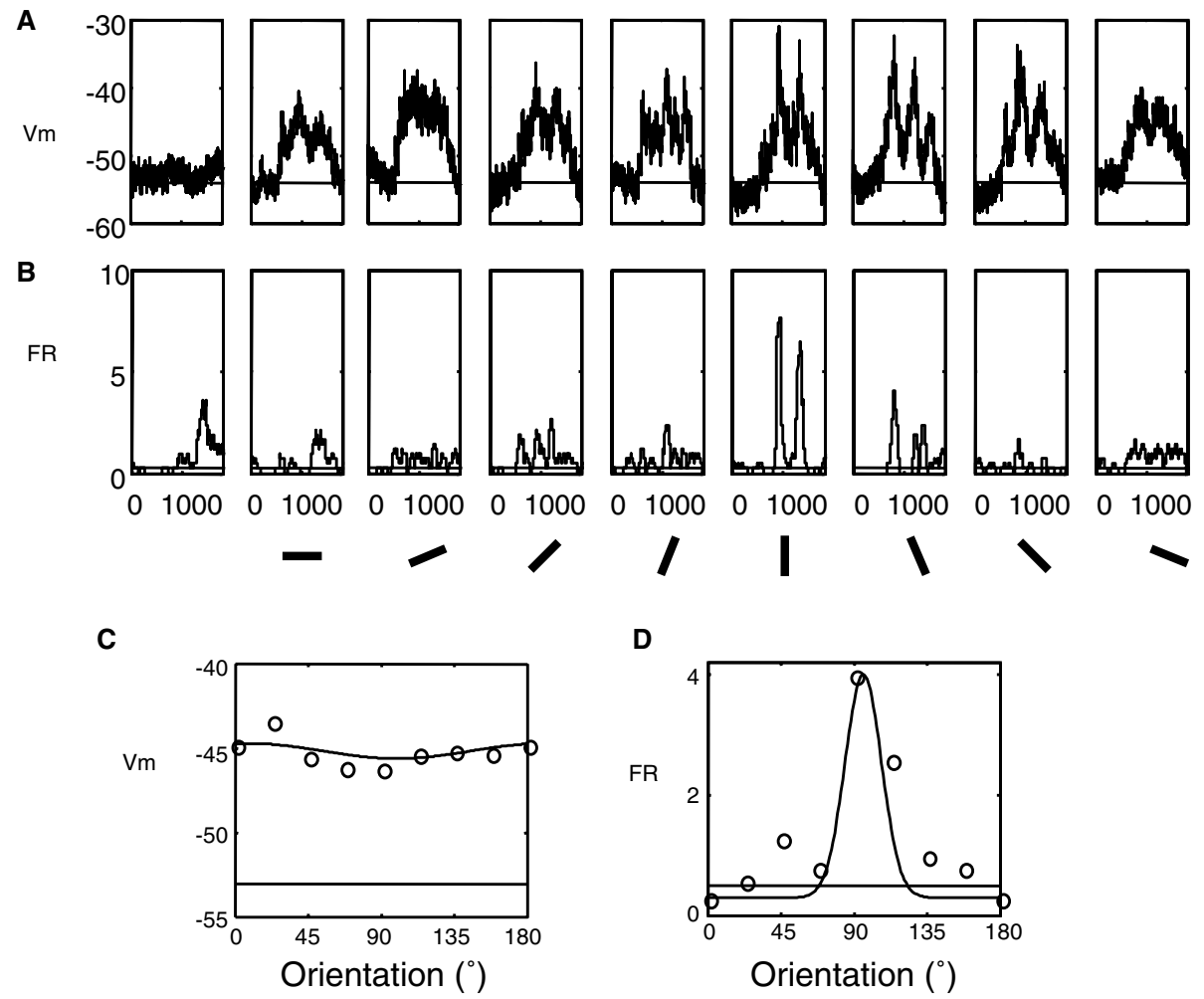

Fig. 3. Membrane potential and firing rate tuning of a simple cell located near a pinwheel center. A. Membrane potential traces during stimulation with each of eight stimulus orientations, centered on the preferred orientation of the cell. All conventions are the same as in Fig. 2. B. PSTH plot of the spike response, extracted from the membrane potential responses in A. C,D. Tuning curves of the membrane potential (Vm) and firing rate (FR).

A

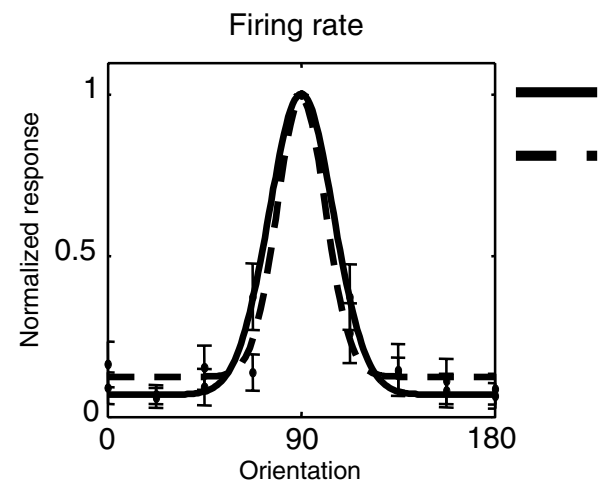

B

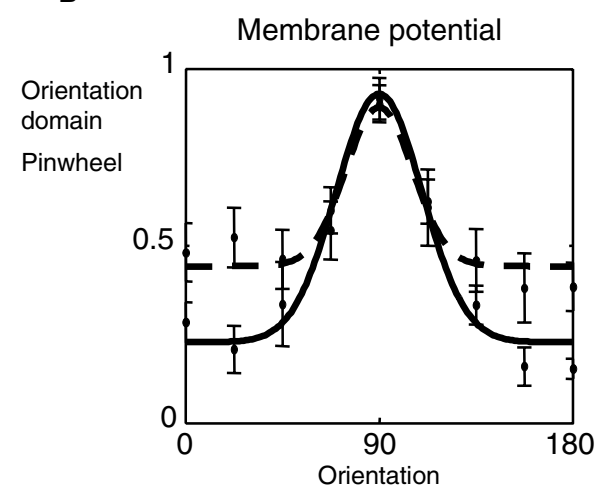

Fig. 4. Tuning curves of pinwheel center neurons have large DC offset. A. Average firing rate tuning curves of a population of $n=11$ pinwheel center cells (dashed line) and $n=7$ orientation domain cells (solid line). The tuning curves of individual neurons were normalized by first aligning the preferred orientation to $90^{\circ}$, subtracting the response to the blank stimulus, and dividing by the response to the preferred orientation. B. Average tuning curves for the same population of pinwheel center and orientation domain neurons as in A. The tuning curves of individual neurons were normalized just as the FR tuning curves before averaging (in this case, the response to the blank stimulus is an estimate of the resting potential, rather than the spontaneous FR). Adapted from [60].

calculate the local input tuning is based on slow intrinsic signals that do not provide information about stimulus phase. A faster method of mapping, such as with voltage sensitive dyes, may provide information about phase-specific cortical inputs. As the F1 modulation of the membrane potential contributes strongly to firing, it will be important to define the source of the inputs contributing to this aspect of the membrane potential response.

\subsection{Mechanisms regulating spike tuning near pinwheel centers}

As described above, the input-output transformation, from synaptic inputs to spiking outputs, is dramatically different in neurons located near pinwheel centers. Inputs coming at orthogonal orientations are essentially filtered out from the spiking response of the neuron. What 
mechanisms might be involved in this process? The synaptic inputs to a neuron are integrated by some linear or nonlinear process, resulting in a net membrane depolarization at the soma. This depolarization may then lead to spiking if the spike threshold is reached. Several groups have examined the factors that determine the transformation of membrane potential into spiking in V1 neurons in vivo. While some studies suggest that a linear model can describe the transformation reasonably well [12], there are indications that the situation may be more complicated. Spike generation has been shown to be dependent on the temporal microstructure of membrane potential fluctuations. High frequency fluctuations in membrane potential lead to higher firing rates, whereas spike rates are lower for the same mean membrane potential without rapid fluctuations $[5,6,71,72]$. Furthermore, the membrane potential contains more high frequency fluctuations in response to the preferred orientation than in response to non-preferred orientations $[6,72]$. In fact, the tuning curve of high frequency components of the membrane potential are more similar to the firing rate tuning curves than are the tuning curves of other slower components of the subthreshold response. Thus, the spiking response can be preferentially enhanced for the preferred orientation by a network or cellular mechanism that enhances rapid membrane potential fluctuations. It will be interesting to see whether such a mechanism is involved in the dramatic sharpening seen in pinwheel center neurons.

It is also possible that the balance of excitation and inhibition plays an important role in regulating the tuning of spiking outputs of neurons near pinwheel centers. There is substantial evidence for shunting inhibition in V1 neurons $[1,10,30,44]$, though the tuning of such inhibition is not entirely clear $[1,44]$. Shunting inhibition can effectively clamp the membrane potential, which has the effect of reducing the influence of excitatory synaptic inputs, and also reducing the variability of membrane potential [44]. Thus if there is sufficient inhibition at non-preferred orientations, the membrane potential may be effectively prevented from reaching threshold, despite the large depolarizations seen in neurons near pinwheel centers. There is evidence that a substantial portion of $\mathrm{V} 1$ neurons receive shunting inhibition that is tuned orthogonal to the preferred orientation of the cell, and such neurons have been hypothesized to be located near pinwheel centers [44]. However, to act as a regulator of firing rate tuning, inhibition need not be orthogonally tuned. So long as the inhibition at non-preferred orientations is adequately strong, it can serve to prevent firing by balancing excitatory inputs. In fact, it has been shown that the membrane potential responses of pinwheel center neurons contain a large component of inhibition [60]. Fig. 5A show the membrane potential responses of a pinwheel center neuron in response to the preferred and orthogonal orientation. The responses were recorded in the presence of three levels of constant current injection. Positive current injection (top traces) unmasks inhibitory inputs, whereas negative current injection (bottom traces), enhances the visibility of the excitatory components. The middle traces show the response at the resting potential. In this condition, there is a substantial depolarization in response to the orthogonal grating. When the cell is hyperpolarized with negative current, the response grows larger, and when the cell is depolarized, the response inverts to hyperpolarization, indicative of inhibition. This suggests that the response to the orthogonal grating is a compound response, composed of both excitation and inhibition. Therefore it seems likely that inhibition, by balancing excitation may play an important role in minimizing the spike response at orthogonal orientations. For comparison, a neuron in an orientation domain (Fig. 5B) shows neither depolarization nor hyperpolarization in response to the orthogonal grating. The results from this cell indicate that orientation domain neurons receive very little, if any, synaptic drive at the orthogonal orientation. This rules out the possibility that the small membrane potential response to the orthogonal orientation in orientation domain neurons results from balanced or shunting inhibition. Although the data are not sufficient to evaluate a quantitative model of in the integration of inhibitory synaptic inputs, these data hint that the integration of inhibitory inputs also follows a spatially isotropic rule. If so, inhibitory inputs may play a crucial role in filtering out responses to non-preferred orientations in pinwheel neurons.

\subsection{Pattern adaptation-induced orientation shifts at pinwheel centers}

At a first glance it is surprising that neurons near pinwheel centers show such sharp spike tuning, given the broadly tuned inputs they receive. It seems that the spike tuning must be tightly regulated by some cortical mechanism, such that the inputs at non-preferred orientations, substantial though they may be, remain below spike threshold. Though the exact cellular mechanisms that provide this regulation are not entirely understood, the most likely explanation is that the levels of excitation and inhibition are balanced either anatomically, by synaptic weighting mechanisms, or cellular integration properties, relative to the spike threshold (see above). For neurons in orientation domains, this balance need not be precise, because there is simply not enough excitation at non-optimal orientations to exceed the spike threshold. However, in many neurons near pinwheel centers, the mean depolarization at orthogonal orientations is very similar to that for the preferred orientation. In such cases, the regulatory mechanisms must be extremely precise in order to maintain sharp spike tuning. In fact, it seems likely that synaptic weights must be dynamically balanced in order to maintain sharp tuning in the context of ongoing activity during the course of normal ongoing function. One might expect, therefore that the tuning curves of neurons near pinwheel centers would be more labile. That is to say, perturbations that alter the strength of synaptic inputs coding orientations on the 
A

Pinwheel
B
Orientation Domain

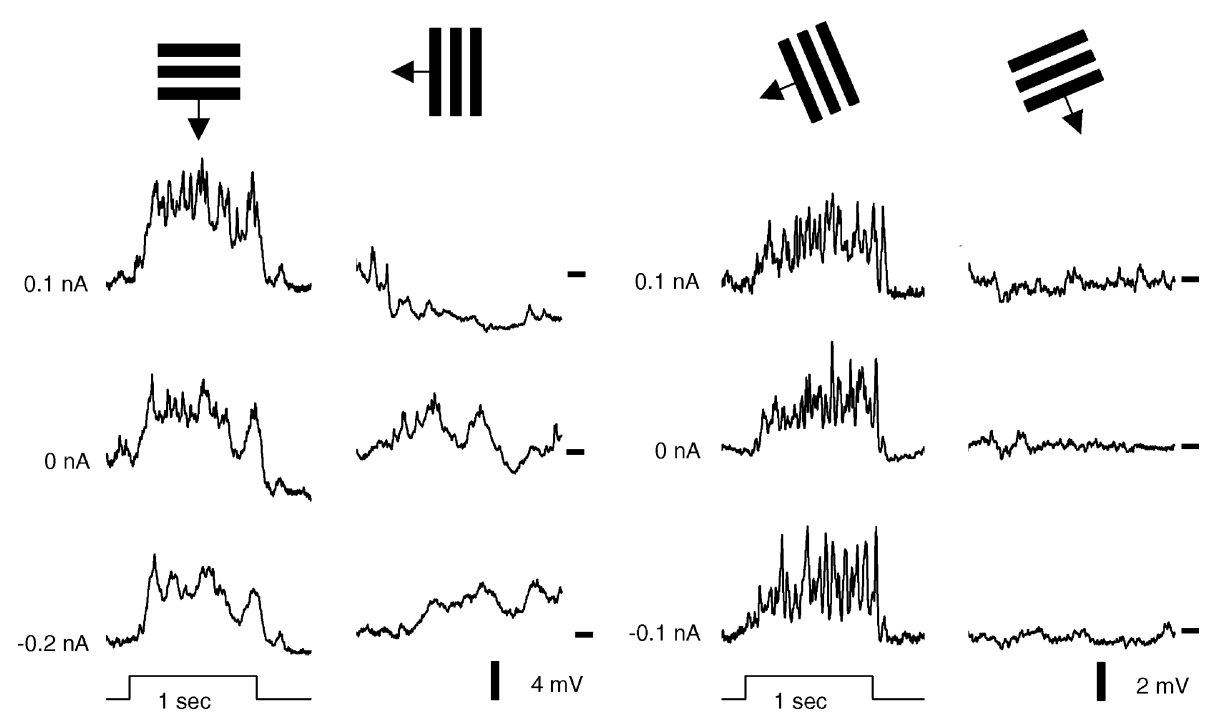

Fig. 5. Pinwheel center neurons receive both excitation and inhibition at the orthogonal orientation. A. Responses of a pinwheel center neuron to the preferred and orthogonal orientation under three levels of membrane depolarization. The left column shows the membrane potential response to the preferred orientation when the cell was depolarized with positive current injection (top), at resting membrane potential (middle), and hyperpolarized with negative current injection (bottom). The right column shows the responses to the orthogonal orientation under with the same current injection protocol. The response at rest and depolarized potentials is mostly depolarization, but the recording with positive current injection reveals a hyperpolarization, indicative of an inhibitory component of the response. B. Responses of an orientation domain neuron under the same conditions used for the pinwheel neuron shown in A. All conventions are identical to those in A. Note that this neuron shows no membrane potential response for the orthogonal orientation, regardless of the manipulation of the membrane polarization, indicating that this neuron does not receive visually-driven synaptic inputs in response to the orthogonal orientation. Adapted from [60].

flanks of the tuning curve, where the inputs are close to threshold, should be able to change the shape of the tuning curve.

Indeed, this is exactly what experiments show [19,21]. When neurons near pinwheel centers are subjected to a pattern adaptation protocol, in which a stimulus of one orientation is presented to the receptive field of a neuron for a period of seconds to minutes, the tuning curve is altered (Fig. 6). Furthermore, it is altered in a predictable way: it shifts away from the orientation of the adapting stimulus. Thus, the cell responds less to orientations near the adapting orientation, and responds more to orientations away from it. This is presumably because the neuron pool representing the adapting orientation has reduced its response ("adapted"), and is therefore providing less drive to the recorded cell. In contrast, neurons located far from pinwheel centers show much smaller shifts in orientation tuning as a result of the pattern adaptation protocol. Overall, neurons near pinwheel centers are over four times more likely to show significant shifts, and the magnitude of the shifts is nearly three times larger. This behavior can be captured by the same model that was used to predict the selectivity of the subthreshold inputs to V1 neurons as a function of map location ([60]; see above). The orientation selectivity of the orientation representation in the local cortical pool presumed to be connected with the recorded neuron was calculated from an isotropic region of the orientation preference map surrounding the recording sites
(Fig. 6A-E). There was a strong inverse correlation between the magnitude of the orientation shift and the orientation selectivity of the local cortical representation [19].

The changes induced with the adaptation protocol involve commensurate changes in the response magnitude to a broad range of stimulus orientations. This suggests that the response of an individual V1 neuron to any particular orientation is a reflection of the adaptation state of the pool of locally projecting neurons representing all orientations. Of course in this highly artificial adaptation paradigm, the adaptation state of many neurons is systematically shifted to a large extent. Under more naturalistic conditions, however, neurons throughout the circuit sit along a continuum of states that can change on multiple timescales, following multiple rules. Recent work has demonstrated changes in response tuning on both short and long timescales. Neural tuning can briefly change in response to very recent stimulation history. Dragoi et al. [20] showed that even brief periods of adaptation, on the timescale of single visual fixations during the scanning of natural scenes, can lead to changes in orientation tuning. These dynamic changes involve changes in both the location of the tuning curve peak as well as the tuning width. Muller et al. [45] have also found evidence for changes in tuning curves resulting from brief periods of pattern adaptation. Thus, orientation tuning is likely to be dynamically shifting on short timescales, in response to the types of stimuli likely to be seen during active vision. 
A
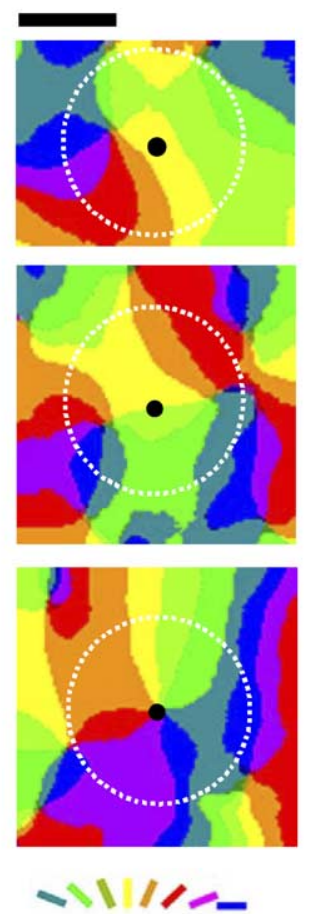

D

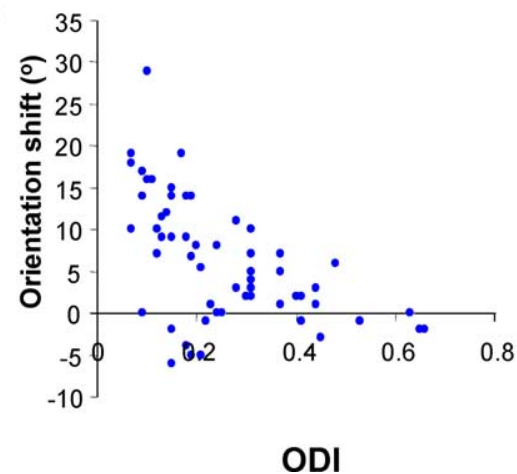

B
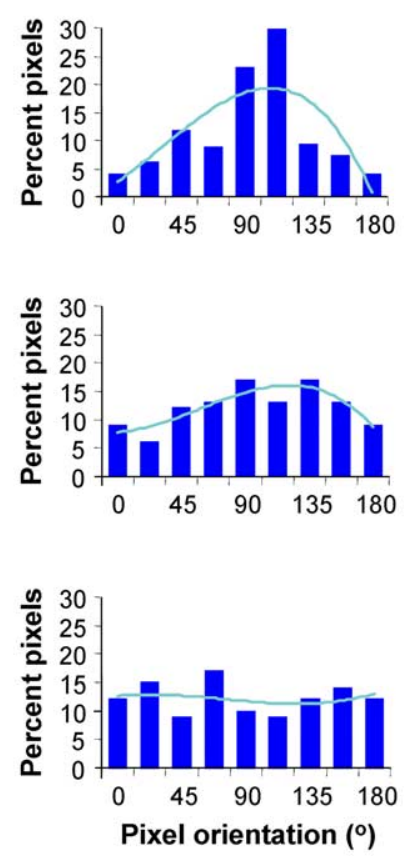

C
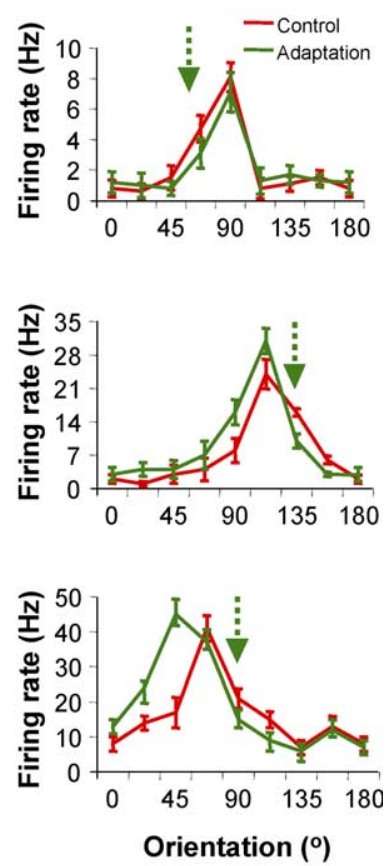

E

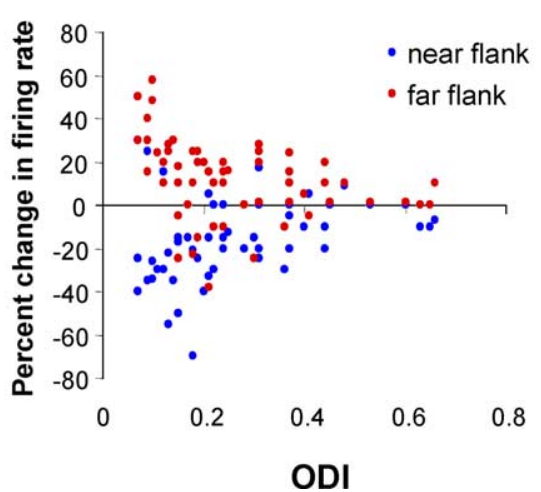

Fig. 6. Adaptation-induced tuning curve shifts depend on the orientation composition of the local cortical circuit. A,B. Orientation map locations (A), and histograms of the orientation composition of the putative local input region (B), estimated from the circles shown in A, for three neurons. C. Tuning curves for three neurons, recorded from the locations indicated in A, before (blue) and after (red) the pattern adaptation protocol. The top neuron, with homogeneous local inputs, shows no shift in tuning, whereas the bottom neuron, located near a pinwheel center, shows a clear shift away from the adapting orientation (green arrows). D. Scatter plot, demonstrating that neurons located in regions of the orientation map with more local heterogeneity (as quantified by the orientation distribution index, ODI) show much larger tuning curve shifts after the adaptation paradigm. E. Scatter plot of the change in magnitude of responses to stimulus orientations near to the adapting orientation (blue), and orientations, on the far flank of the tuning curve (red), far from the adapting orientation. Responses to near orientations are suppressed, whereas responses to far orientations are enhanced, demonstrating that the tuning curve shifts result from an active process, rather than a simple diminution of responses near the adapting orientation. Adapted from [19].

There is also evidence, from several recent studies, of more prolonged changes in tuning characteristics, resulting from more long-term, repetitive stimulus patterns. Using a stimulus protocol known to induce synaptic plasticity in vitro, Yao and Dan [75] found that a conditioning paradigm can induce shifts in the tuning curves of V1 neurons lasting $\sim 30$ minutes. Using an electrical stimulation paradigm, Schuett et al. [59] demonstrated that the structure of the orientation preference map can also be reliably shifted for periods up to several hours. There is also recent data to suggest that sensory learning paradigms in awake animals can change tuning curve properties of V1 neurons [58]. Thus, there is evidence that there are complex changes in the tuning curve properties on multiple timescales that are continually changing the state of the activity across the cortical network. Only in the context of the circuit as a whole can the variability and dynamics of the responses of individual neurons make sense.

There is direct evidence that the current state of the cortical network can have a dramatic influence on the responses of individual neurons. Using fast imaging of population membrane potential with voltage sensitive dyes, 
it has been demonstrated that cortical networks often spontaneously approach states similar to orientation maps $[3,35]$. Furthermore, the variability of responses of individual cells is in part attributable to cortical states across broad regions of the network [4]. The spontaneous activity of neurons can also reflect the functional circuit of which the neuron is a part $[3,35]$.

\subsection{Computational models of orientation tuning}

As described above, there is mounting evidence that there is substantial diversity in the orientation tuning properties of V1 neurons. This fact poses significant problems for most models of the synaptic mechanisms responsible for orientation tuning. Most models try to produce an "average" V1 tuning curve, rather than the whole range of tuning curves observed in V1 neurons. These models are generally created to describe the mechanism for orientation tuning - the underlying assumption is that there is low variability in the orientation tuning properties. Correspondingly, the orientation tuning of the inputs to all neurons is generally identical. But, as discussed above, the inputs vary depending on location in the orientation map. Recently, models have been proposed that explicitly incorporate the pinwheel structure of the orientation map with spatially isotropic inputs $[34,43]$. Such models replicate several of the features of the tuning curves seen experimentally, including a broad range of tuning sharpness and variability in the role of inhibition in sharpening tuning at different map locations. As more studies describe the details of which tuning characteristics are variable, and which are constant, across different cortical locations, such models will be important in constraining our thinking about the mechanisms involved in shaping cortical responses. In this way, models can play an important role in bridging the gap between individual neurons and network interactions, which can be difficult without a clear analytical framework.

\section{Discussion and conclusions}

The goal of this review has been to highlight recent work aimed at understanding the response properties of individual neurons within the context of their cortical network.

We have proposed a simple model of the inputs to V1 neurons to estimate the functional characteristics of the inputs to different sites in the orientation preference map. The model proposes that, regardless of map position, a large portion of the inputs to V1 neurons arise from a small isotropic region of cortical space. This model is supported by anatomical data, and provides a good explanation of the experimental data.

This model is of course over-simplified. As mentioned above, there may be dramatic differences in the nature and integration of inputs in different cortical layers. Neurons in each layer receive a distinct complement of inputs, and so it is not surprising that there are marked differences in the orientation tuning properties from layer to layer [53]. However, these differences have been averaged out in the analysis presented here. On one hand, such simplification highlights the robustness of the differences seen despite the smearing that comes with averaging, and on the other hand this points out that more work needs to be done to understand the complexities of synaptic integration in the cortex.

The influence of the local map structure on subthreshold responses is an important step towards understanding the impact of surrounding cortical networks on the response properties of individual V1 neurons. However, as mentioned above, there are many other factors that can shape the nature and integration of inputs by individual neurons. New approaches that combine imaging techniques to describe the behavior of large circuits with single neuron recordings have been demonstrated to be an important starting point in creating a more comprehensive integrated description of orientation tuning in V1 at several levels of description. As imaging techniques are refined, and potentially new methods, perhaps incorporating genetic tools are developed, the different levels of description may be brought even closer together.

The simplified model of integration of inputs relative to the orientation preference map outlined above will need to be elaborated to incorporate many other features of the functional organization in V1. One prominent simplification is that we have only considered orientation preference, at the expense of many other stimulus features to which V1 neurons are tuned. V1 neurons are selective for retinal position, eye of presentation, spatial frequency, direction of movement, disparity, length, and perhaps more stimulus features. Of these, there are orderly maps of at least retinotopy, ocular dominance, spatial frequency, and direction $[77,78]$. Some of these are likely to be emergent cortical phenomena (direction, length), whereas others are clearly delineated by the pattern of thalamocortical afferents (retinotopy, ocular dominance). Regardless of these aspects, each of these features must be encoded, to some extent, in the spatial pattern of synaptic inputs. It will therefore be interesting to test how these tuning parameters relate to local circuits. In fact, some of the variance that is unexplained by our simple circular model of orientation integration probably relates to the unknown position of our neurons in these other circuits. Ultimately, one would like to construct a model of the functional wiring rules along all of these dimensions. Presumably, the subthreshold response of any V1 neuron to any arbitrary stimulus could be well predicted by its position in the circuits that compute each of these (and maybe more) features.

\section{References}

[1] J.S. Anderson, M. Carandini, D. Ferster, Orientation tuning of input conductance, excitation, and inhibition in cat primary visual cortex, $\mathrm{J}$. Neurophysiol. 84 (2000) 909-926. 
[2] A. Angelucci, J.B. Levitt, E.J. Walton, J.M. Hupe, J. Bullier, J.S. Lund, Circuits for local and global signal integration in primary visual cortex, J. Neurosci. 22 (2002) 8633-8646.

[3] A. Arieli, D. Shoham, R. Hildesheim, A. Grinvald, Coherent spatiotemporal patterns of ongoing activity revealed by real-time optical imaging coupled with single-unit recording in the cat visual cortex, J. Neurophysiol. 73 (1995) 2072-2093.

[4] A. Arieli, A. Sterkin, A. Grinvald, A. Aertsen, Dynamics of ongoing activity: explanation of the large variability in evoked cortical responses, Science 273 (1996) 1868-1871.

[5] R. Azouz, C.M. Gray, Dynamic spike threshold reveals a mechanism for synaptic coincidence detection in cortical neurons in vivo, Proc. Natl. Acad. Sci. USA 97 (2000) 8110-8115.

[6] R. Azouz, C.M. Gray, Adaptive coincidence detection and dynamic gain control in visual cortical neurons in vivo, Neuron 37 (2003) 513523.

[7] R. Ben-Yishai, R.L. Bar-Or, H. Sompolinsky, Theory of orientation tuning in visual cortex, Proc. Natl. Acad. Sci. USA 92 (1995) 3844 3848 .

[8] G.G. Blasdel, Orientation selectivity, preference, and continuity in monkey striate cortex, J. Neurosci. 12 (1992) 3139-3161.

[9] T. Bonhoeffer, A. Grinvald, Iso-orientation domains in cat visual cortex are arranged in pinwheel-like patterns, Nature 353 (1991) 429 431.

[10] L.J. Borg-Graham, C. Monier, Y. Fregnac, Visual input evokes transient and strong shunting inhibition in visual cortical neurons, Nature 393 (1998) 369-373.

[11] W.H. Bosking, Y. Zhang, B. Schofield, D. Fitzpatrick, Orientation selectivity and the arrangement of horizontal connections in tree shrew striate cortex, J. Neurosci. 17 (1997) 2112-2127.

[12] M. Carandini, D. Ferster, Membrane potential and firing rate in cat primary visual cortex, J. Neurosci. 20 (2000) 470-484.

[13] J.M. Crook, U.T. Eysel, Gaba-induced inactivation of functionally characterized sites in cat visual cortex (area 18): effects on orientation tuning, J. Neurosci. 12 (1992) 1816-1825.

[14] J.M. Crook, Z.F. Kisvarday, U.T. Eysel, Gaba-induced inactivation of functionally characterized sites in cat striate cortex: effects on orientation tuning and direction selectivity, Vis. Neurosci. 14 (1997) $141-158$.

[15] J.M. Crook, Z.F. Kisvarday, U.T. Eysel, Evidence for a contribution of lateral inhibition to orientation tuning and direction selectivity in cat visual cortex: reversible inactivation of functionally characterized sites combined with neuroanatomical tracing techniques, Eur. J. Neurosci. 10 (1998) 2056-2075.

[16] A. Das, C.D. Gilbert, Topography of contextual modulations mediated by short-range interactions in primary visual cortex, Nature 399 (1999) 655-661.

[17] R.J. Douglas, C. Koch, M. Mahowald, K.A. Martin, H.H. Suarez, Recurrent excitation in neocortical circuits, Science 269(1995) 981-985.

[18] R.J. Douglas, K.A. Martin, A functional microcircuit for cat visual cortex, J. Physiol. 440 (1991) 735-769.

[19] V. Dragoi, C. Rivadulla, M. Sur, Foci of orientation plasticity in visual cortex, Nature 411 (2001) 80-86.

[20] V. Dragoi, J. Sharma, E.K. Miller, M. Sur, Dynamics of neuronal sensitivity in visual cortex and local feature discrimination, Nat. Neurosci. 5 (2002) 883-891.

[21] V. Dragoi, J. Sharma, M. Sur, Adaptation-induced plasticity of orientation tuning in adult visual cortex, Neuron 28 (2000) 287-298.

[22] U.A. Ernst, K.R. Pawelzik, C. Sahar-Pikielny, M.V. Tsodyks, Intracortical origin of visual maps, Nat. Neurosci. 4 (2001) 431-436.

[23] D. Ferster, Orientation selectivity of synaptic potentials in neurons of cat primary visual cortex, J. Neurosci. 6 (1986) 1284-1301.

[24] D. Ferster, S. Chung, H. Wheat, Orientation selectivity of thalamic input to simple cells of cat visual cortex, Nature 380 (1996) 249-252.

[25] D. Ferster, K.D. Miller, Neural mechanisms of orientation selectivity in the visual cortex, Annu. Rev. Neurosci. 23 (2000) 441-471.

[26] C.D. Gilbert, Laminar differences in receptive field properties of cells in cat primary visual cortex, J. Physiol. 268 (1977) 391-421.
[27] C.D. Gilbert, A. Das, M. Ito, M. Kapadia, G. Westheimer, Spatial integration and cortical dynamics, Proc. Natl. Acad. Sci. USA 93 (1996) 615-622.

[28] C.D. Gilbert, T.N. Wiesel, Clustered intrinsic connections in cat visual cortex, J. Neurosci. 3 (1983) 1116-1133

[29] C.D. Gilbert, T.N. Wiesel, Functional organization of the visual cortex, Prog. Brain Res. 58 (1983) 209-218.

[30] J.A. Hirsch, J.M. Alonso, R.C. Reid, L.M. Martinez, Synaptic integration in striate cortical simple cells, J. Neurosci. 18 (1998) $9517-$ 9528.

[31] J.A. Hirsch, C.A. Gallagher, J.M. Alonso, L.M. Martinez, Ascending projections of simple and complex cells in layer 6 of the cat striate cortex, J. Neurosci. 18 (1998) 8086-8094.

[32] D.H. Hubel, T.H. Wiesel, Receptive fields, binocular interaction and functional architecture of the cat's visual cortex, J. Physiol. 160 (1962) $106-154$

[33] M. Hubener, D. Shoham, A. Grinvald, T. Bonhoeffer, Spatial relationships among three columnar systems in cat area $17, \mathrm{~J}$. Neurosci. 17 (1997) 9270-9284.

[34] K. Kang, M. Shelley, H. Sompolinsky, Mexican hats and pinwheels in visual cortex, Proc. Natl. Acad. Sci. USA 100 (2003) 2848-2853.

[35] T. Kenet, D. Bibitchkov, M. Tsodyks, A. Grinvald, A. Arieli, Spontaneously emerging cortical representations of visual attributes, Nature 425 (2003) 954-956.

[36] Z.F. Kisvarday, E. Toth, M. Rausch, U.T. Eysel, Orientation-specific relationship between populations of excitatory and inhibitory lateral connections in the visual cortex of the cat, Cereb. Cortex 7 (1997) 605-618.

[37] I. Lampl, J.S. Anderson, D.C. Gillespie, D. Ferster, Prediction of orientation selectivity from receptive field architecture in simple cells of cat visual cortex, Neuron 30 (2001) 263-274.

[38] M.S. Livingstone, D.H. Hubel, Anatomy and physiology of a color system in the primate visual cortex, J. Neurosci. 4 (1984) 309356.

[39] R. Malach, Y. Amir, M. Harel, A. Grinvald, Relationship between intrinsic connections and functional architecture revealed by optical imaging and in vivo targeted biocytin injections in primate striate cortex, Proc. Natl. Acad. Sci. USA 90 (1993) 10469-10473.

[40] P.E. Maldonado, I. Godecke, C.M. Gray, T. Bonhoeffer, Orientation selectivity in pinwheel centers in cat striate cortex, Science 276 (1997) $1551-1555$.

[41] K.A. Martin, Microcircuits in visual cortex, Curr. Opin. Neurobiol. 12 (2002) 418-425.

[42] L.M. Martinez, J.M. Alonso, R.C. Reid, J.A. Hirsch, Laminar processing of stimulus orientation in cat visual cortex, J. Physiol. 540 (2002) 321-333.

[43] D. McLaughlin, R. Shapley, M. Shelley, D.J. Wielaard, A neuronal network model of macaque primary visual cortex (v1): orientation selectivity and dynamics in the input layer 4calpha, Proc. Natl. Acad. Sci. USA 97 (2000) 8087-8092.

[44] C. Monier, F. Chavane, P. Baudot, L.J. Graham, Y. Fregnac, Orientation and direction selectivity of synaptic inputs in visual cortical neurons: a diversity of combinations produces spike tuning, Neuron 37 (2003) 663-680.

[45] J.R. Muller, A.B. Metha, J. Krauskopf, P. Lennie, Rapid adaptation in visual cortex to the structure of images, Science 285 (1999) 14051408.

[46] S. Nelson, L. Toth, B. Sheth, M. Sur, Orientation selectivity of cortical neurons during intracellular blockade of inhibition, Science 265 (1994) 774-777.

[47] S.B. Nelson, Cortical microcircuits: diverse or canonical?, Neuron 36 (2002) 19-27.

[48] X. Pei, T.R. Vidyasagar, M. Volgushev, O.D. Creutzfeldt, Receptive field analysis and orientation selectivity of postsynaptic potentials of simple cells in cat visual cortex, J. Neurosci. 14 (1994) 7130-7140.

[49] S.C. Rao, L.J. Toth, M. Sur, Optically imaged maps of orientation preference in primary visual cortex of cats and ferrets, J. Comp. Neurol. 387 (1997) 358-370. 
[50] R.C. Reid, J.M. Alonso, Specificity of monosynaptic connections from thalamus to visual cortex, Nature 378 (1995) 281-284.

[51] D.L. Ringach, M.J. Hawken, R. Shapley, Dynamics of orientation tuning in macaque primary visual cortex, Nature 387 (1997) 281-284.

[52] D.L. Ringach, M.J. Hawken, R. Shapley, Dynamics of orientation tuning in macaque v1: the role of global and tuned suppression, J. Neurophysiol. 26 (2003) 26.

[53] D.L. Ringach, R.M. Shapley, M.J. Hawken, Orientation selectivity in macaque v1: diversity and laminar dependence, J. Neurosci. 22 (2002) $5639-5651$.

[54] K.S. Rockland, J.S. Lund, Intrinsic laminar lattice connections in primate visual cortex, J. Comp. Neurol. 216 (1983) 303-318.

[55] B. Roerig, B. Chen, Relationships of local inhibitory and excitatory circuits to orientation preference maps in ferret visual cortex, Cereb. Cortex 12 (2002) 187-198.

[56] B. Roerig, J.P. Kao, Organization of intracortical circuits in relation to direction preference maps in ferret visual cortex, J. Neurosci. 19 (1999) RC44.

[57] H. Sato, N. Katsuyama, H. Tamura, Y. Hata, T. Tsumoto, Mechanisms underlying orientation selectivity of neurons in the primary visual cortex of the macaque, J. Physiol. 494 (Pt 3) (1996) $757-771$.

[58] A. Schoups, R. Vogels, N. Qian, G. Orban, Practising orientation identification improves orientation coding in v1 neurons, Nature 412 (2001) 549-553.

[59] S. Schuett, T. Bonhoeffer, M. Hubener, Pairing-induced changes of orientation maps in cat visual cortex, Neuron 32 (2001) 325-337.

[60] J. Schummers, J. Marino, M. Sur, Synaptic integration by v1 neurons depends on location within the orientation map, Neuron 36 (2002) 969-978.

[61] J. Schummers, M. Sur, Paper presented at the 30th Annual Meeting of the Society for Neuroscience N.O., LA, 5 November 2000.

[62] J. Sharma, A. Angelucci, M. Sur, Induction of visual orientation modules in auditory cortex, Nature 404 (2000) 841-847.

[63] D. Somers, V. Dragoi, M. Sur, Orientation selectivity and its modulation by local and long-range connections in visual cortex, in: B.R. Payne, A. Peters (Eds.), The Cat Primary Visual Cortex, Academic Press, Boston, 2001, pp. 471-520.

[64] D.C. Somers, S.B. Nelson, M. Sur, An emergent model of orientation selectivity in cat visual cortical simple cells, J. Neurosci. 15 (1995) $5448-5465$.

[65] H. Sompolinsky, R. Shapley, New perspectives on the mechanisms for orientation selectivity, Curr. Opin. Neurobiol. 7 (1997) 514-522.
[66] L.J. Toth, S.C. Rao, D.S. Kim, D. Somers, M. Sur, Subthreshold facilitation and suppression in primary visual cortex revealed by intrinsic signal imaging, Proc. Natl. Acad. Sci. USA 93 (1996) 98699874.

[67] T.W. Troyer, A.E. Krukowski, N.J. Priebe, K.D. Miller, Contrastinvariant orientation tuning in cat visual cortex: thalamocortical input tuning and correlation-based intracortical connectivity, J. Neurosci. 18 (1998) 5908-5927.

[68] M. Tsodyks, T. Kenet, A. Grinvald, A. Arieli, Linking spontaneous activity of single cortical neurons and the underlying functional architecture, Science 286 (1999) 1943-1946.

[69] T.R. Vidyasagar, X. Pei, M. Volgushev, Multiple mechanisms underlying the orientation selectivity of visual cortical neurones, Trends Neurosci. 19 (1996) 272-277.

[70] M. Volgushev, X. Pei, T.R. Vidyasagar, O.D. Creutzfeldt, Excitation and inhibition in orientation selectivity of cat visual cortex neurons revealed by whole-cell recordings in vivo, Vis. Neurosci. 10 (1993) 1151-1155.

[71] M. Volgushev, J. Pernberg, U.T. Eysel, A novel mechanism of response selectivity of neurons in cat visual cortex, J. Physiol. 540 (2002) 307-320.

[72] M. Volgushev, J. Pernberg, U.T. Eysel, Gamma-frequency fluctuations of the membrane potential and response selectivity in visual cortical neurons, Eur. J. Neurosci. 17 (2003) 1768-1776.

[73] M. Volgushev, T.R. Vidyasagar, M. Chistiakova, T. Yousef, U.T. Eysel, Membrane properties and spike generation in rat visual cortical cells during reversible cooling, J. Physiol. 522 (Pt 1) (2000) 59-76.

[74] M. Weliky, K. Kandler, D. Fitzpatrick, L.C. Katz, Patterns of excitation and inhibition evoked by horizontal connections in visual cortex share a common relationship to orientation columns, Neuron 15 (1995) 541-552.

[75] H. Yao, Y. Dan, Stimulus timing-dependent plasticity in cortical processing of orientation, Neuron 32 (2001) 315-323.

[76] T. Yousef, E. Toth, M. Rausch, U.T. Eysel, Z.F. Kisvarday, Topography of orientation centre connections in the primary visual cortex of the cat, Neuroreport 12 (2001) 1693-1699.

[77] H. Yu, B.J. Farley, D.Z. Jin, M. Sur, The coordinated mapping of visual space and response features in visual cortex, Neuron 21 (2005) 267-280.

[78] H.-B. Yu, B.J. Farley, M. Sur, Spatial relationships between retinotopy and four other feature maps in ferret visual cortex, vol. 28, Society for Neuroscience, Orlando, FL, 2 November 2002 . 